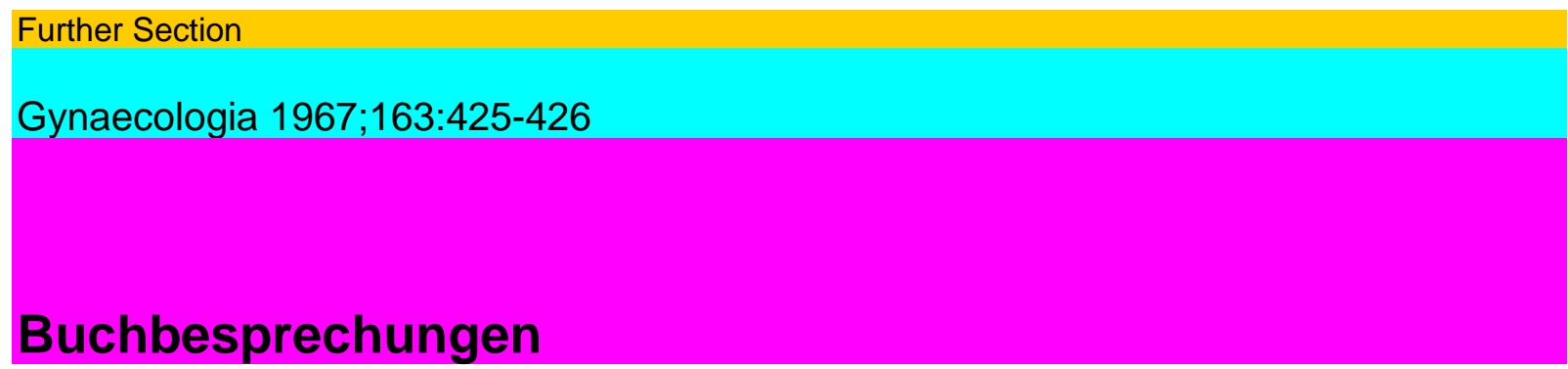

\title{
Book REVIEWS LIVRES NOUVEAUX
}

Brewer, Thomas H.: Metabolic Toxemia of Late Pregnancy. A Disease of Malnutrition. Charles C. Thomas, Springfield 1966. 127 pp.; Price: \$ 8.50.

Der schmale Band mit knapp 130 Seiten beschreibt das Bild einer Schwanger-schaftsgestose, welches wir hier nur äusserst seiten sehen. Es entspricht einer schwe-ren Präeklarnpsie bis Eklampsie. Als Todesursache wird oft die Leberruptur ange-geben.

Auf den Beobachtungen an diesem uns unbekannten Krankengut baut der Autor seine Theorie auf, dass die Gestose ihren Ursprung in einer massiven Fehl- und Unterernährung habe. Durch die dadurch entstehende Hypoalbuminurie werde das Entstehen der Odeme bewirkt und die Abbaufunktion der Leber gestört. Die Leber werde ausserdem durch die vermehrten Detoxikationsanforderungen von seiten des fötalen Stoffwechsel, der vermehrten mütterlichen und placentaren Hormonpro-duktion und der Abbauprodukte des Darminhaltes belastet. Mit der Dekompensation der Leber versucht der Autor das Bild dieser metabolischen Toxikose zu erklären. Die Therapie besteht im wesentlichen in Albumininfusionen und Sterilisierung der Darmflora mit Breitbandantibiotika.

Das Buch ist in einem gut leseriichen, fast journalistischen Stil geschrieben, ohneTabellen und genaue Zahlenangaben. Es ist wohl auch weniger als streng wissen-schaftliches Werk gedacht, eher soil es den Faktor der Schwangerschafts-Ernährungsowie die Bedeutung der Prophylaxe für die Nephropathie in den Vordergrundrücken.

E. T. Ríppmann, Basel

Kríge, Z. F.: Vaginal Hysterectomy and Genital Prolapse Repair. A Contribution to the Vaginal Approach in Operative Gynaecology. Witwatersrand University Press, Johannesburg 1965. 143 pp. 9 fig.; Price: sFr. 32.-.

Der Autor legt seine Erfahrungen über 1540 persönlich vorgenommene vaginale Hysterektomien nieder und schildert die von ihm benutzte Technik. Nach einer lesenswerten historischen Einleitung und der Besprechung der Aetiologie von Genital-prolapsen wird die vom Autor entwickelte operative Technik niedergelegt. Sie be-ruht auf einer möglichst funktionellen Wiederherstellung der Beckenbodenverhält-nisse, welche folgende Punkte besonders berücksichtigt:

Enterozelen sind ein ausserordentlich häufiger Nebenbefund von eminenter Wichtigkeit, indem ihre Nichtbeachtung zu den anatomisch schlechten Spätresul-taten führt.

Vaginale Hysterektomien ohne Kolporrhaphie sind, im Gegensatz zu der An-sicht vieler kontinentaleuropäischer Operateure, nur in den seltensten Fallen indiziert.

Im Gegensatz zu englischen Autoren ist eine vaginale totale Hysterektomie mit alleiniger vorderer Kolporrhaphie ungenügend. Die operative Technik unter-scheidet sich von anderen dadurch, dass auf eine Infiltration der vorderen und hin-teren Vaginalwand verzichtet wird (klarer anatomische Verhältnisse). Zweitens, dass die Peritonisierung mit einer Tabaksbeutelnaht von sacral her durchgeführt wird und an diese Naht zusätzlich die Ligamenta sacro-uterina fixiert werden. Drit-tens wird die Fascia pubo-cervicalis an das hintere Scheidengewölbe fixiert und die vordere Kolporrliaphie mit einer obligatorischen modifizierten Stoeckelschen Naht beendet. Als 
letztes wird die hintere Kolporrhaphie sehr hoch begonnen. Der Zeit-aufwand für die ganze Operation beträgt nach Angabe des Autors etwa 45 Minuten.

426 Buchbesprechungen - Book Reviews - Livres Nouveaux

Die postoperativen Resultate scheinen besser zu sein als diejenigen anderer Au-toren, jedoch geht nicht hervor, wie weit die Nachkontrollen wirklich Spätresultate sind.

Die Monographie ist sehr leicht zu lesen und bietet dem operativ tätigen Gynä-kologen auf alle Fälle eine Bereicherung seines Wissens. Obwohl die Abbildungen zur Operationstechnik besser sein dürften, ist das Buch zu empfehlen.

W. Obolensky, Liestal

Crawford, J. S.: Grundlagen und Praxis der geburtshilflichen Anaesthesie. 2. Auflage 1965. VEB Verlag Volk und Gesundheit, Berlin. XV+ 384 S.; 25 Abb.; Preis: MDN 48,50.

Es ist dies die zweite Auflage der deutschen Übersetzung von "Principles and Practice of Obstetric Anaesthesia”.

H. F. Poppelbaum hat mehr als nur Ubersetzungsarbeit geleistet. In neun über-sichtlichen Kapiteln werden alle Belange der geburtshilflichen Anaesthesie wie folgtbehandelt: 1. Mutter, 2. Placenta, 3. Placenta, Pharmaka, Betus, 4. Analgesie, 5. Schnitt- und Zangen-Entbindung, 6. andere Anaesthesieindikatíon, 7. Kind, 8. Ausrüstung und Organisation.

Nach jedem Kapitel findet sich eine fast unerschöpfliche Literaturangabe. In einpaar Kapiteln nimmt der Verfasser eine bewusst dogmatische Haltung ein. Damiterhält das Buch auch einen guten kritischen Charakter. Es werden sowohl die inEuropa als auch die in den USA

gebräuchlichen Methoden aufgeführt. Das Buchsollte als Handbuch in der Bibliothek eines jeden Geburtshelfers und Anaesthesistenstehen. E. Meyer, Liestal

COMING MEDICAL MEETINGS KONGRESSKALENDER TABLEAU DES CONGRÈS

Mannheim: Die nächste Tagung der Deutschen Gesellschaft für Zytologie, ge-meinsam mit der Österreichischen Gesellschaft für angewandte Zytologie, findet vom 19.-21. Oktober 1967 statt. Auskunft: Privatdozent Dr. H.-J. Soost, Maistrasse 11 (I. Universitäts-Frauenklinik), D-8 München 15.

Rostock-Warnemünde: Vom 29. 5. bis 1. 6. 1968 findet in Rostock-Warnemünde ein Gynäkologen-Kongress, veranstaltet von der Gesellschaft für Geburtshilfe und Gynäkologie der DDR, statt. Wissenschaftliche Leitung: Prof. Dr. H. Kyank; Organisatorische Leitung: Doz. Dr. R. Schwarz.

A. Anmeldung von Vorträgen bis zum 30. November 1967 unter Beifügung eines Kurzreferates erbeten an Prof. Dr. Kyank, Universitäts-Frauenklinik Rostock, Doberaner Strasse 142, D-25 Rostock 1. 